\title{
Evidence of an irradiated accretion disc in XTE J1818-245
}

\author{
J. A. Zurita Heras ${ }^{1}$, S. Chaty ${ }^{1}$, M. Cadolle Bel ${ }^{2}$ and L. Prat ${ }^{1}$
}

${ }^{1}$ AIM Paris Saclay, CEA-DSM/CNRS-INSU/Université Paris 7 Denis Diderot, IRFU/Service d'Astrophysique, Bât. 709 L'Orme des Merisiers, 91191 Gif-sur-Yvette, France email: juan-antonio.zurita-heras@cea.fr, sylvain.chaty@cea.fr, lionel.prat@cea.fr

${ }^{2}$ ESAC, ISOC, Villanueva de la Cañada, Madrid, Spain email: Marion.Cadolle@sciops.esa.int

\begin{abstract}
The X-ray transient source XTE J1818-245 went through an outburst in 2005 that was observed during a multi-wavelength campaign from radio to soft $\gamma$-rays. We performed new optical observations with the ESO/NTT telescope at La Silla. The broad-band spectral energy distribution revealed that the outer parts of the accretion disc had to be irradiated by its inner parts to explain the optical emission.
\end{abstract}

Keywords. X-rays: binaries, X-rays: individual (XTE J1818-245).

\section{Introduction}

XTE J1818-245 was discovered at high-energies with $R X T E$ during a bright outburst that started on August 12, 2005 (Levine, Swank, Lin, et al. 2005). Cadolle Bel, Prat, Rodriguez, et al. (2009) performed several observations (from radio to soft $\gamma$-rays) from August to September and concluded that the new X-ray transient source is probably a low-mass X-ray binary and a black hole candidate, located closer to us than the Galactic centre. We carried out new optical observations with the ESO $3.6 \mathrm{~m}$ New Technology Telescope at La Silla Observatory on August 24, 2005, when the X-ray flux was still decaying, and when the source was probably in a soft-intermediate state.

\section{Spectral energy distribution}

Each subset of data (radio/optical/X-rays/hard X-rays) reported in the SED displays different segments, each approximately power law in shape with different spectral indices. We focus our interest to the optical emission where different components of the system may contribute: the outer part of the accretion disc, the companion star, and the jet.

Model 1: Viscously heated accretion disc. The high-energy spectrum of XTE J1818-245 is represented by a combination of an accretion disc modelled with a multi-colour disc, a broad iron emission line at 6-7 keV, and a hard component modelled with a Comptonisation model. The fit of this model 1 (gaussian+ezdiskbb+compTT in Xspec), reproduces correctly the high-energy data with a reduced $\chi^{2}$ of $\chi_{\nu}^{2}=1.21$ with 29 degrees of freedom (dof). However, model 1 fails to reproduce both visible+HE data, particularly the model being a factor 10 below the visible points $\left(\chi_{\nu}^{2}=1.45\right.$ with 34 dof, see Fig. 1 left $)$. We also added other components to the model to take into account the presence of the secondary star (temperature of $5000 \mathrm{~K}$, radius of $1 R_{\odot}$ and distance of $3.5 \mathrm{kpc}$ ) and a possible contribution in the visible from a jet (flat power law normalised to the $8.4 \mathrm{GHz}$ flux). This stellar model gives an emission compatible with the upper limits derived from 2MASS, USNO and REM. The sum of all these different components fails to reproduce the optical data that show a steeper shape with a spectral index $\alpha=1.15$ (in $F_{\nu} \sim \nu^{\alpha}$ ). 

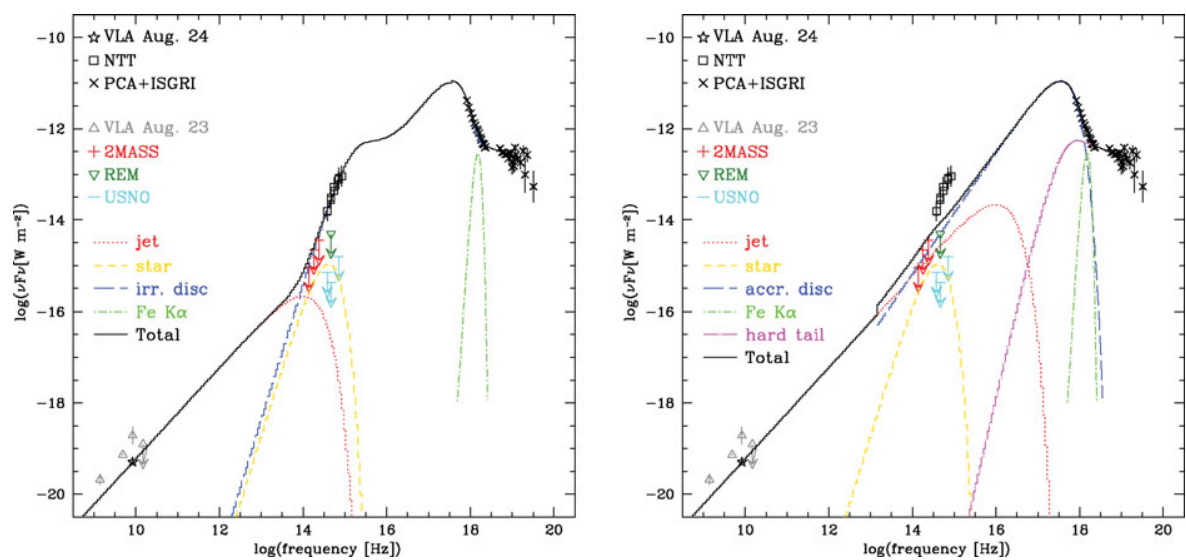

Figure 1. Broad-band SED of XTE J1818-245 using simultaneous VLA(Aug. 24)/NTT/ PCA/ISGRI data. We also show non-simultaneous data from VLA(Aug. 23)/2MASS/REM/ USNO. left: Model 1; right: Model 2.

Model 2: Irradiated accretion disc. Irradiation of the outer parts of the accretion disc by soft X-rays has been proposed to explain the UV-optical emission in soft X-ray transients (Hynes, Haswell, Chaty, et al. 2002). We considered an irradiated disc model that takes into account the emission of the disc, the emission of the Compton component, and both the irradiation of the inner part of the disc by the Compton tail and that of the outer part by the inner part (diskir, Gierliński, Done, \& Page 2008,2009, and references therein for a discussion about the irradiation). We fitted the optical+HE data with model 2 (gaussian+diskir), reproducing well the data with $\chi_{\nu}^{2}=1.11$ (see Fig. 2 right). The addition of the stellar component and the contribution of the jet in the visible does not improve the fit. The emission of the jet may contribute to the redder part of the optical emission, however we cannot assess this statement without simultaneous radio-infrared observations. The Comptonisation-to-disc ratio of XTE J1818-245 is low with $L_{\mathrm{c}} / L_{\mathrm{d}}=0.07_{-0.01}^{+0.03}<<1$ as expected when the soft component in X-rays is important. Meanwhile, its irradiation fraction is $f_{\text {out }}=2.4_{-0.8}^{+1.2} \times 10^{-3}$ which is $2 \times f_{\text {out }}$ of XTE J1817-330 in the intermediate state. The absolute determination of this fraction is uncertain by a factor 2 , but a change by a factor 6 between the soft and hard states has been observed in other X-ray transients (Gierliński, Done, \& Page 2009). Thus, the irradiation fraction of XTE J1818-245 is compatible with that of XTE J1817-330 when they are in a similar state. We also derive an inner radius of $R_{\text {in }}=(31 \pm 9) \mathrm{km}\left(\sim 6 \times R_{\mathrm{g}}\right.$ for a $3.5 M_{\odot}$ black hole with $\left.R_{\mathrm{g}} \equiv G M / c^{2}\right)$, thus an outer radius of $R_{\text {out }} \sim 4 \times 10^{10} \mathrm{~cm}$ which is in agreement with Cadolle Bel, Prat, Rodriguez, et al. (2009).

\section{References}

Cadolle Bel, M., Prat, L., Rodriguez, J., Ribó, M., Barragán, L., D’Avanzo, P., Hannikainen, D. C., Kuulkers, E., Campana, S., Moldón, J., Chaty, S., Zurita Heras, J., Goldwurm, A., \& Goldoni, P. 2009, A\&A, 501, 1

Gierliński, M., Done, C., \& Page, K. 2008 MNRAS, 388, 753

Gierliński, M., Done, C., \& Page, K. 2009 MNRAS, 392, 1106

Hynes, R. I., Haswell, C. A., Chaty, S., Shrader, C. R., \& Cui, W. 2002 MNRAS, 331, 169

Levine, A. M., Swank, J. H., Lin, D., \& Remillard, R. A. 2005, ATel, 578 